Review

\title{
STAT3: An Anti-Invasive Factor in Colorectal Cancer?
}

\section{Petrus Rudolf de Jong ${ }^{1}$, Ji-Hun Mo ${ }^{2}$, Alexandra R. Harris ${ }^{1}$, Jongdae Lee ${ }^{1, *}$ and Eyal Raz ${ }^{1}$}

1 Department of Medicine, University of California, San Diego, 9500 Gilman Dr. MC 0663, La Jolla, CA 92093, USA; E-Mails: pdejong@ucsd.edu (P.R.J.); arh5ru@virginia.edu (A.R.H.); eraz@ucsd.edu (E.R.)

2 Department of Otorhinolaryngology, Dankook University College of Medicine, 16-5 Anseo-dong, Cheonan, Chungcheongnam-do 330-715, Korea; E-Mail: jihunmo@gmail.com

* Author to whom correspondence should be addressed; E-Mail: j142lee@ucsd.edu; Tel.: +1-619-543-3579; Fax: +1-619-543-5247.

Received: 5 March 2014; in revised form: 13 June 2014 / Accepted: 20 June 2014 / Published: 3 July 2014

\begin{abstract}
Signal Transducer and Activator of Transcription 3 (STAT3) is activated in a majority of cancers, and promotes tumorigenesis and even metastasis through transcriptional activation of its target genes. Recently, we discovered that STAT3 suppresses epithelial-to-mesenchymal transition (EMT) and thus metastasis in a mouse model of colorectal cancer (CRC), while it did not affect the overall tumor burden. Furthermore, we found that STAT3 in intestinal epithelial cells (IEC) suppresses EMT by regulating stability of an EMT inducer, SNAI-1 (Snail-1). Here, STAT3 functions as an adaptor rather than a transcription factor in the post-translational modification of SNAI-1. In this review, we discuss the unexpected and contradictory role of STAT3 in metastasis of CRC and its clinical implications.
\end{abstract}

Keywords: signal transducer and activator of transcription 3 (STAT3); colorectal cancer (CRC); metastasis; epithelial to mesenchymal transition (EMT); Snail-1 (SNAI-1); glycogen synthase kinase $3 \beta$ (GSK3 $\beta$ ); Snail; adenomatous polyposis coli (APC); tight junction (TJ); matrix metalloproteinases (MMPs) 


\section{Colorectal Cancer (CRC)}

CRC is the second most frequent cancer, after lung cancer, that leads to death in the US and Europe [1]. The development of sporadic human CRC is driven by the accumulation of somatic mutations in oncogenes (gain-of-function) and/or tumor-suppressor genes (loss-of-function), as well as epigenetic changes. Three etiological subtypes of genomic instability in CRC have been described, including chromosomal instability (CIN), microsatellite instability (MSI), and its related phenomenon known as the $\mathrm{CpG}$ island methylator phenotype (CIMP).

CIN is the most common cause of CRC (80\%-85\%) and is associated with a poor prognosis [2]. CIN in CRC is characterized by chromosomal rearrangements (unbalanced translocation, deletion) and abnormality in chromosome numbers (gain or loss of whole chromosomes) [3]. Additionally, CIN is caused by somatic mutations in genes that regulate the mitotic spindle checkpoint, DNA replication checkpoints, DNA damage checkpoints, chromosome metabolism, or centrosome function [2,4]. The CIN phenotype is used to measure intercellular heterogeneity in chromosome number and describe cancers with aneuploidy, polyploidy, or loss of heterozygosity ( $\mathrm{LOH}$ ) [5]. Gain or loss of the chromosomal copy number contributes to development and/or progression of cancer via inactivation of tumor suppressor genes, activation of oncogenes, or change in gene dosage (increasing or decreasing expression of these genes) [6]. In fact, copy number alterations seem to be the major mechanism for transcriptional deregulation of cancer genes in CRC [7].

Loss of function of mismatch-repair (MMR) genes (MLH1, MSH2, MSH6, and PMS2) is responsible for the epiphenomenon of MSI, which is associated with mutations in tumor-suppressor genes. CIMP involves epigenetic silencing of tumor suppressor genes, such as MLH1, due to aberrant DNA methylation (promoter hypomethylation of oncogenes or hypermethylation of tumor suppressor genes). This oncogenic mechanism shows overlap with MSI in CRC subgroups [8]. MSI occurs in $\sim 15 \%$ of CRC patients, and $\mathrm{MSI}^{+}$CRC has a better prognosis when compared with $\mathrm{CIN}^{-}$or $\mathrm{MSI}^{-}$ CRC. Approximately $20 \%$ of CRC patients are CIMP ${ }^{+}$.

Whereas $\mathrm{MSI}^{+} \mathrm{CIMP}^{+} \mathrm{CRC}$ confers a better prognosis for CRC, patients with $\mathrm{MSI}^{-} \mathrm{CIMP}^{+} \mathrm{CRC}$ have a worse prognosis [9]. Recently, De Sousa e Melo et al. [10] confirmed the existence of multiple CRC subtypes by gene expression profiling. They identified three categories, including colon cancer subtypes that correspond to the $\mathrm{CIN}^{+}$or MSI/CIMP ${ }^{+}$class, respectively. In addition, they identified a third subclass that was characterized by hallmarks of epithelial-mesenchymal transition (EMT), shared features with sessile serrated adenoma, and conferred a poor prognosis in CRC [10]. Sadanandam et al. proposed an alternative classification system based on gene expression profiling of primary CRC tumors [11]. These novel CRC subtypes included enterocyte, goblet-like, transit-amplifying, inflammatory, and stem-like tumor cells, correlating with their colon-crypt location, Wnt activity, and response to adjuvant or metastatic treatment [11]. In addition to their prognostic features, a higher resolution of CRC subtypes allows for better stratification of CRC patients in clinical trials, and may better predict their response to targeted therapy. Importantly, despite all these novel insights, the molecular events that lead to tumor invasion and metastasis are still largely unknown.

With regard to oncogenic pathways, CIN is associated with inactivating mutations in the APC (adenomatous polyposis coli) gene or, to a lesser extent, gain-of-function mutations in the CTNNB1 ( $\beta$-catenin) gene. These mutations result in the formation of an early adenoma. This is followed by the 
adenoma-to-carcinoma transition (in CIN) through sequential genetic mutations in oncogenes (e.g., KRAS, PIK3CA, PTEN, or EGFR/ERBB family members) and/or tumor suppressor genes (e.g., TP53, $S M A D 4, B A X)[12,13]$. While germline mutations in the $A P C$ gene are responsible for familial adenomatous polyposis (FAP), somatic mutations in APC occur in $\sim 90 \%$ of sporadic colorectal tumors. These mutations in APC lead to unrestricted activation of $\beta$-catenin, which in turn activates many genes responsible for tumorigenesis, such as MYC. In fact, an integrated genetic analysis by The Cancer Genome Atlas (TCGA) Network showed that nearly 100\% of CRC tumors showed changes in transcriptional targets of MYC [13].

Most of CRC-related mortality is due to metastasis, which develops in at least $50 \%$ of CRC patients, and the majority of metastatic tumors are not surgically resectable [14]. Thus, it is critical to understand the mechanisms underlying CRC metastasis.

The most widely used animal model of CRC is the $A p c^{\min /+}$ mouse strain in which multiple intestinal neoplasia (min) spontaneously develop, mostly in the small intestine. Adenomas in this model generally do not progress to adenocarcinomas. Nonetheless, this model has been an important tool to understand tumorigenesis and metastasis of CRC. We made a conditional deletion of STAT3 in IEC of these mice (Apc ${ }^{\text {in/+ }} / S_{\text {Stat }}{ }^{I E C-K O}$ mice) to investigate the role of STAT3 in CRC and found an anti-carcinogenic role for STAT3 [15], which was unexpected given a plethora of literature supporting the pro-oncogenic role of STAT3. Musteanu et al. also reported this anti-invasive role for STAT3 [16]. Together, these data suggest that STAT3 suppresses malignant transformation of adenomas in the $A p c^{\min /+}$ mouse strain.

\section{STAT3 Signaling and Its Role in Cancers}

STAT3 plays an essential role in a variety of physiological functions, including development, proliferation, and immune defense [17-20]. STAT3 is phosphorylated on a tyrosine residue (Tyr-705) by an upstream kinase, JAK2 (Janus kinase 2). In addition to phosphorylation at Tyr-705, STAT3 is phosphorylated at Ser-727 and acetylated at Lys-685 [21], which is thought to be required for its full transcriptional activity. The phosphorylated protein forms either a homodimer or a heterodimer with other STAT proteins, then translocates to the nucleus and transcribes target genes. However, STAT3 can also regulate expression of certain target genes (e.g., RANTES, IL-6, IL-8, Met, and M-Ras) in a phosphorylation-independent manner [22,23]. Several non-transcriptional functions of STAT3 have also been reported. STAT3 functions as an adaptor protein, connecting IFNAR1 (interferon $\alpha$ receptor 1) and the p85 regulatory subunit of PI3K (phosphoinositide 3-kinase) [24], and it inhibits stathmin that depolymerizes microtubules [25]. Activated STAT3, through interaction with phosphorylated paxillin and focal adhesion kinase (FAK), was shown to promote ovarian cancer cell invasiveness [26].

Numerous in vitro and in vivo studies indicate that STAT3 promotes tumorigenesis of a variety of cancers, thus it is generally recognized as an oncogene. STAT3 is activated in $70 \%$ of all solid and hematological tumors $[27,28]$, and it was found activated in $72 \%$ of colorectal carcinomas but only in 18\% of colorectal adenomas [29]. Furthermore, p-STAT3 expression is associated with a poor prognosis in CRC, independent of MSI, CIMP, BRAF, or KRAS mutation status [30]. STAT3 is rarely mutated in cancer cells but rather activated by upstream signals, such as receptor tyrosine kinses (RTKs), mutated JAKs, or oncogenic cellular tyrosine kinases (CTKs), such as Src. Recently, somatic mutations in the SH2 dimerization and activation domain of STAT3 were discovered in $40 \%$ of 
patients with large granular lymphocytic leukemia, and these mutations were associated with enhanced phosphorylation of STAT3 and its localization in the nucleus [31].

\section{STAT3 in Intestinal Epithelial Cells (IEC) Promotes Cell Survival and Epithelial Barrier Function}

Membranes of IEC, like any epithelium, are polarized with tight junctions as the boundary. The apical membrane, facing the lumen, expresses distinctive proteins (e.g., digestive enzymes, nutrient transporters, etc.) in IEC. Conversely, proteins in the basolateral membrane resemble those in other unpolarized cell types [32]. This is achieved by complex and coordinated protein sorting and targeting machinery [33]. We have shown that TLR9 is expressed in both the apical and basolateral membranes, but only apical TLR9 generates a response that suppresses the inflammatory responses of basolateral TLRs, including TLR3, TLR5, and TLR9 [32]. This was surprising for three main reasons. First, since TLR9 is expressed in endosomes of immune cells, our data indicate that localization of TLR9 is not determined by its primary sequence. Second, it indicates that different downstream effectors of the same receptor (TLR9) are utilized depending on the location of the receptor. Whether this is unique to TLR9 remains to be seen. Third, when IEC lose polarity (by culturing on a plastic surface), TLR9 signaling mainly resembles that of the basolateral TLR9 (inflammatory). We suspect that intracellular signaling cascades in IEC could be altered under pathological conditions where polarity is disrupted (e.g., inflammation or cancer). Recently, we found such an example: interferon $\alpha$ (IFN $\alpha$ ) activates STAT3 preferentially in polarized IEC, while it activates both STAT1 and STAT3 to the same extent in unpolarized IEC (Figure 1A). STAT1 is rapidly degraded by the proteasome upon IFN $\alpha$ stimulation in polarized IEC, which can be rescued by blockade of the proteasome (Figure 1B). Regulation of STAT1 signaling by polarization in IEC resulted in a distinctive pattern of gene expression upon IFN $\alpha$ stimulation (Figure 1C): more inflammatory genes, such as IRF-1 and STAT1, were induced in unpolarized IEC, whereas anti-apoptotic genes, such as Bcl-2, were induced in polarized IEC. SLIM, a ubiquitin ligase of STAT1 [34], was also induced by IFN $\alpha$ in polarized IEC, which is consistent with the result that STAT1 is degraded by the proteasome under this condition. Thus, from these results and other evidence in the literature, it was expected that STAT3 in IEC should promote tumorigenesis by preventing apoptosis.

However, we also found that STAT3 promotes polarization of IEC, measured by trans-epithelial electrical resistance (TER), and reduces trans-epithelial permeability, a measure of barrier function (Figure 2). Since carcinomas typically lose polarity before metastasis, our data also suggest that STAT3 might inhibit EMT and thus metastasis of CRC.

\section{STAT3 Does Not Affect Intestinal Tumor Initiation}

Contrary to the expectation that STAT3 would be a major driver of tumorigenesis but consistent with the data by Musteanu et al. [16], we found that STAT3 in IEC plays only a minor role in tumor initiation in the $A p c^{\mathrm{min} /+}$ model. However, both groups observed a dramatic increase in tumor invasion of $A p c^{\mathrm{min} /+}$ tumors in the intestines upon deletion of Stat3 in IEC in vivo. It must be noted that some of our observations in the $A p c^{\min /+} / S_{t a t} 3^{I E C-K O}$ strain differ from those reported by Musteanu et al [16]. Whereas they observed a slight reduction in tumor multiplicity at a late stage [15], we found that the number of adenomas in the small and large intestine did not significantly change in $\mathrm{ApC}^{\mathrm{min} /+} / \mathrm{Stat}^{I E C-K O}$ 
mice [15]. Furthermore, they observed an increased rate of IEC proliferation and nuclear translocation of $\beta$-catenin in tumors of $A p c^{\mathrm{min} /+} /$ Stat $3^{I E C-K O}$ mice [16], whereas we found no differences in these parameters [15]. Interestingly, Musteanu et al. [16] observed increased p-STAT3 levels in Apc ${ }^{\mathrm{min} /+}$ adenomas [16], and we found that total STAT3 expression in adenomas was substantially higher than that in normal IEC (unpublished data). These data both suggest that STAT3 is highly activated in tumor cells, since STAT3 activates its own transcription [35]. In the Apc ${ }^{\mathrm{min} /+}$ model, $\beta$-catenin is the driver of tumorigenesis and STAT3 has been directly implicated in the activation of $\beta$-catenin. For example, the expression of a dominant negative (DN)-STAT3 or pharmacological inhibition of JAK/STAT3 signaling induced translocation of $\beta$-catenin from the nucleus to the cytoplasm. This resulted in reduced transcriptional activity of $\beta$-catenin and inhibition of cell proliferation [36]. In contrast, Musteanu et al. proposed that STAT3 ${ }^{\mathrm{IEC}-\mathrm{KO}}$ resulted in down-regulation of the cell adhesion protein, CEACAM1, with a concomitant increase in nuclear $\beta$-catenin [16]. However, we did not observe this change in localization of $\beta$-catenin and therefore propose an alternative model in which STAT3 negatively regulates EMT.

Figure 1. STAT3 is preferentially activated by IFN $\alpha$, while STAT1 is degraded by the proteasome in polarized IEC. (A) Polarized or unpolarized HCA-7 cells were stimulated with IFN $\alpha(10 \mathrm{ng} / \mathrm{mL})$ for the indicated time period and the indicated proteins were measured by immunoblotting; (B) Polarized HCA-7 cells were stimulated with IFN $\alpha$ (10 ng/mL) for the indicated time period in the absence or presence of MG132 (10 $\mu \mathrm{M})$ and the indicated proteins were measured by immunoblotting. To detect ubiquitination of STAT1, STAT1 was immunoprecipitated and blotted with anti-ubiquitin antibody; (C) Polarity-dependent gene activation by IFN $\alpha$ in IECs. Polarized (P) or unpolarized (UP) HCA-7 cells were stimulated with IFN $\alpha(10 \mathrm{ng} / \mathrm{mL})$ for $1 \mathrm{hr}$ and the induction of indicated genes by IFN $\alpha$ was measured by qPCR. The data represents one of two independent experiments with similar results.

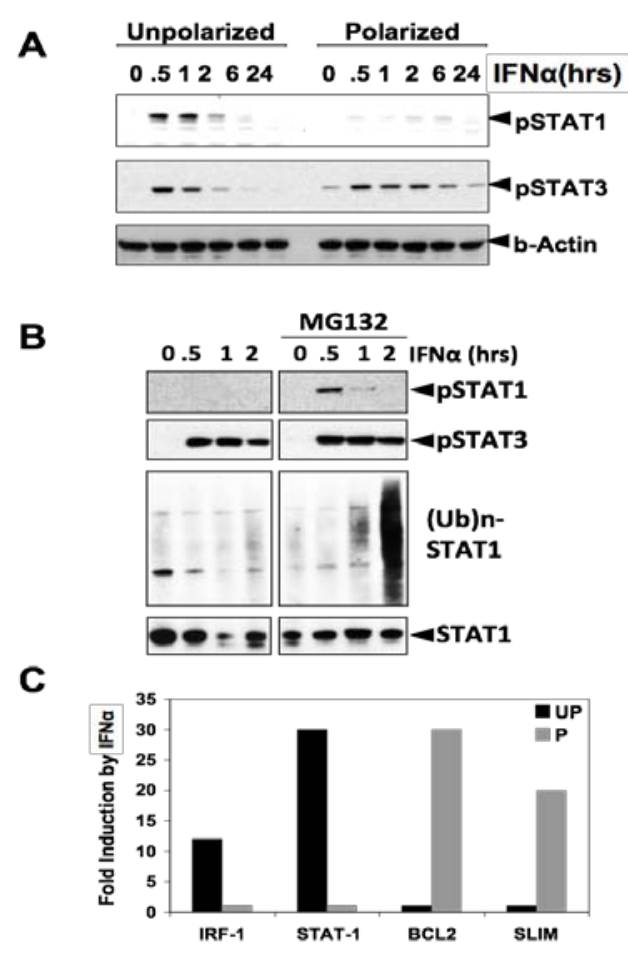


Figure 2. STAT3 promotes polarization and barrier function of IEC in vitro and in vivo. (A) STAT3 in HCA-7 cells was silenced by RNAi and the indicated proteins were measured by immunoblotting; (B) Cells transfected with the indicated siRNA were plated on a Transwell plate and transepithelial electrical resistance (TER) was measured at the indicated time. $(n=6)$ (* represents $p$ value $<0.05$, ANOVA); (C) The barrier function of HCA-7 cells was assessed by using FITC-sulfonic acid (FS). FS was measured five days after transfection $(n=6)$; (D) The deletion of STAT3 ${ }^{\mathrm{IEC}}$ in mice was confirmed by immunohistochemistry (IHC) as described [37]. STAT3 staining (brown) in the KO mice is confined to mononuclear cells in the LP (Lamina Propria) but is absent in IEC; (E) Intestinal permeability of KO mice is significantly higher than that of WT mice $(p=0.001)$. Mice were gavaged with FITC-dextran (FD, MW 4000, $6 \mathrm{mg} / \mathrm{mouse}$ ) and the level of FD in serum was measured 90 min post administration.

A

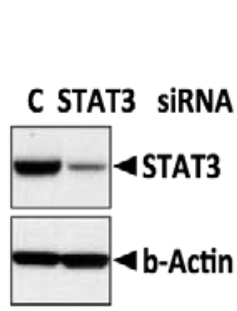

B

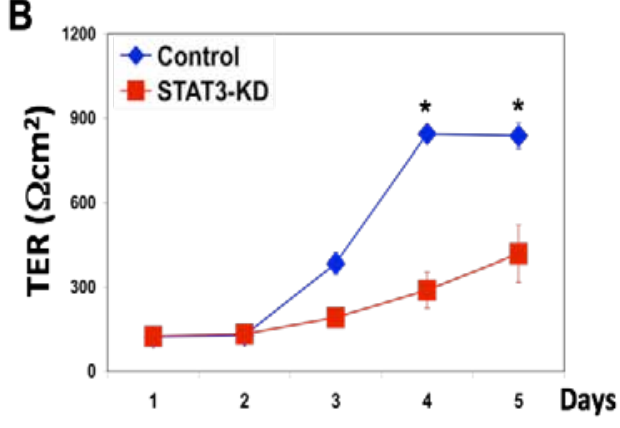

D

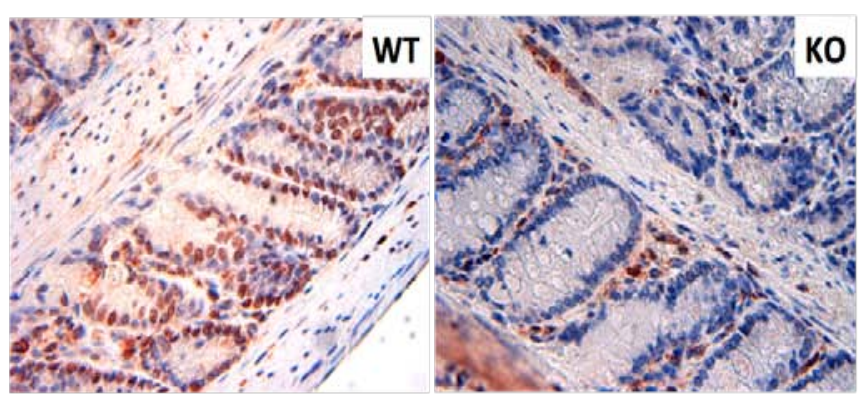

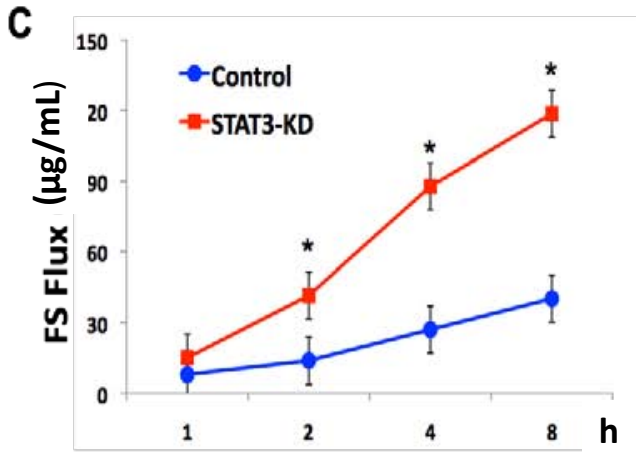

$E$

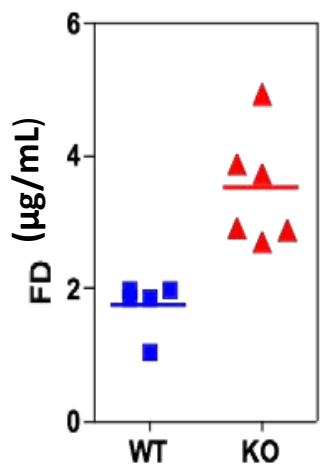

It is important to note that STAT3 is essential for tumorigenesis in the colitis-associated cancer (CAC) model in mice [38,39]. This model employs a carcinogen (azoxymethane) to induce mutagenesis, which is followed by repeated administration of DSS (dextran sulfate sodium) to induce chronic colitis [40]. Metastasis in this model is still dependent on mutations in the $\beta$-catenin gene [41]. Although dysfunctional APC can affect molecules other than $\beta$-catenin, $\beta$-catenin is essential for intestinal tumorigenesis. How does STAT3 play such a contradictory role in sporadic versus colitis-induced CRC? We found that STAT3-deficient IEC are highly sensitive to DSS-induced death (unpublished data in Stat3 ${ }^{I E C-K O}$ mice), as reported by Grivennikov et al. [38]. It is possible that potential STAT3-deficient cancer cells in this model may not have survived the repeated DSS treatments in the CAC model. The $A p c^{\min /+}$ model is not driven by the carcinogenic effects of inflammation or any other chemical interventions. Thus, the effect of epithelial STAT3 signaling on tumor initiation is likely very different in sporadic CRC versus CAC. 


\section{STAT3 Suppresses Adenoma to Adenocarcinoma Transition}

While tumorigenesis was only minimally affected by deletion of STAT3, tumors in $A p c^{\text {min/+ }} / \mathrm{Stat}^{\mathrm{IEC}-\mathrm{KO}}$ mice invaded deeply into the mucosal stroma, submucosa, and muscle, indicating that STAT3 suppresses metastasis [15,16]. Epithelial-to-mesenchymal transition (EMT) is an integral process of metastasis and generally considered a prerequisite [42-45]. Epithelial cells are tightly interlocked with each other by junctional complexes, including tight junctions (TJ) and adherens junctions (Figure 1). Loss of junctional proteins such as E-cadherin, an adherens junction protein, is a hallmark of EMT and metastasis in many cancers [46-48]. Cancer cells that acquired a mesenchymal phenotype express prototypical mesenchymal proteins, such as vimentin and fibronectin, as well as various metalloproteinases. STAT3 is reportedly involved in IL-6-induced EMT in head and neck tumor metastasis [49], and promotes metastasis of melanoma to the brain by induction of the extracellular matrix-degrading metalloproteinases, including MMP-2 and MMP-9 [50].

In contrast, we found that STAT3 plays an opposite role in the $A p c^{\min /+}$ model. Intestinal adenomas in $A p c^{\text {min/+ }}$ mice did not show down-regulation of E-cadherin, while there was a remarkable down-regulation of the TJ protein, CLDN-3 (claudin-3) when compared to normal IEC [51]. Furthermore, expression of CLDN-3 and CLDN-5 in tumor cells was markedly decreased in tumor cells of $A p c^{\mathrm{min} /+} /$ Stat $3^{\text {IEC-KO }}$ mice compared with those in $A p c^{\mathrm{min} /+}$ mice; there was no difference in E-cadherin or occludin levels. More importantly, all tumors in $A p c^{\min /+} / S t a t 3^{I E C-K O}$ mice expressed high levels of vimentin and, in some tumors, fibronectin, whereas those in $A p c^{\mathrm{min} /+}$ mice rarely expressed either [15]. Results showing the loss of TJ proteins [51] and slight elevation of vimentin [52] suggest that a low grade of EMT is initiated in $\mathrm{Apc}^{\mathrm{min} /+}$ mice and indicate that STAT3 deletion can accelerate this process. As discussed above, Musteanu et al. proposed that STAT3 impairs invasion of IEC tumors via the cell adhesion molecule, CEACAM1 [16]. Therefore, STAT3 appears to be intimately involved in cell adhesion of IEC and other epithelial cell types.

Expression of activated STAT3 in colon cancer cells (HT-29 or CoGa-1) induced metalloproteinases MMP1, MMP3, MMP7, and MMP9, which were demonstrated to aid invasiveness of these cells [53]. Our data showed that, while MMP9 and MMP15 (MT2-MMP) expression in tumor cells was negligible in both $A p c^{\mathrm{min} /+}$ and $A p c^{\mathrm{min} /+} /$ Stat $3^{\text {IEC-KO }}$ mice, MMP7 expression in tumor cells was significantly lower in $A p c^{\mathrm{min} /+} /$ Stat $3^{I E C-K O}$ mice. These data indicate that MMP7, MMP9, and MMP15 are not likely to contribute to tumor invasion in these mice. However, MMP14 (MT1-MMP) expression in tumor cells was highly elevated in $A p c^{\min /+} / S_{t a t} 3^{I E C-K O}$ mice but undetectable in $A p c^{\min /+}$ mice. Since MMP14 is particularly efficient in hydrolyzing basement membranes [54,55], it may be involved in tumor invasion in $A p c^{\text {min/+ }} /$ Stat $^{I E C-K O}$ mice. Furthermore, overexpression of the MT1-MMP gene is reported as a useful predictor of outcomes in patients with CRC [56]. Several MT1-MMP inhibitors are under development for cancer therapy [57].

\section{STAT3 Inhibits Tumor Invasion via Regulation of an EMT Inducer SNAI-1}

EMT is induced by a group of transcription repressors, including SNAI-1, Slug, Zeb-1, Zeb-2, Twist, E47, and KLF. These factors directly or indirectly repress transcription of E-cadherin and other junction proteins, including claudins and desmosomes, thereby facilitating EMT [42-45]. Among the tested EMT inducers, only SNAI-1 was significantly elevated in tumors of $\mathrm{Apc}^{\mathrm{min} /+} / \mathrm{Stat}^{\mathrm{IEC}-\mathrm{KO}}$ 
mice [15]. Silencing STAT3 in a few human CRC cell lines also induced expression of SNAI-1 but not other EMT inducers, while overexpression of STAT3-WT suppressed SNAI-1 expression. STAT3 knockdown significantly increased the invasiveness of a CRC cell line, HCT116, which was completely dependent on SNAI-1 [15]. Our data are directly contradictory to a previous study in which STAT3 reportedly increased the invasiveness of HT-29 or CoGa-1 [53]. HCT116 cells are APC ${ }^{W T}$, whereas both HT-29 and CoGa-1 cell lines carry APC mutations. This suggests that the suppression of EMT by STAT3 is not dependent on constitutive activation of Wnt signaling. Furthermore, Xiong et al. reported that STAT3 mediates down-regulation of E-cadherin through Zeb-1, thereby promoting EMT and the invasive properties of CRC [58], which is also contradictory to our findings. Their observations were mainly done in human CRC lines SW1116 and LoVo [58], which were derived from a primary tumor and metastasized CRC, respectively, and carry APC mutations. Finally, another report suggested that STAT3 inhibition negatively regulated the migratory and invasive properties of HCT116 cells [59]. Differential in vitro conditions may cause STAT3 to have opposite effects on EMT, even within the same cell line, an issue that remains to be clarified. The tumor microenvironment, which is lost in CRC cell line cultures, dictates the metastatic capacity of disseminated CRC cells in vivo. In this regard, the GP130/STAT3 signaling axis has been shown to contribute to CRC metastasis [60]. Thus, STAT3 displays both pro- and anti-metastatic effects in CRC, the outcome of which may be determined by the tumor microenvironment.

\section{STAT3 Coordinates SNAI-1 Stability as a Molecular Adaptor}

Unexpectedly, STAT3 deletion or knockdown in IEC did not significantly affect the transcription of SNAI [15], indicating that STAT3 regulates SNAI-1 at a post-transcriptional level. SNAI in a CRC cell line was constitutively ubiquitinated and degraded by proteasomes. STAT3 knockdown increased the level of SNAI-1 but diminished the level of SNAI ubiquitination [15], indicating that STAT3 facilitates ubiquitination of SNAI-1. Phosphorylation of SNAI-1 by GSK3 $\beta$ at two different sites regulates the fate of SNAI-1 during EMT: phosphorylation of the first motif induces ubiquitination of SNAI-1, whereas phosphorylation of the second motif controls its subcellular localization [61]. As expected, we found that pharmacological inhibition or knockdown of GSK3 $\beta$ elevated the SNAI-1 level in a CRC cell line [15]. STAT3 and GSK3 $\beta$ co-immunoprecipitated, but expression of STAT3 protein was not affected by GSK3 $\beta$ or vise versa. However, whether STAT3 has to be phosphorylated to interact with GSK3 $\beta$ is still to be determined. STAT3 knockdown elevated phosphorylation on Ser9 of GSK3 $\beta$, which inhibits its kinase activity [62]. GSK3 $\beta$ is also involved in activation of $\beta$-catenin, but GSK3 $\alpha$ can compensate for the absence of GSK3 $\beta$ [63]. This may explain why there were no changes in $\beta$-catenin levels. Ectopic expression of a constitutively active GSK3 $\beta$ mutant in a CRC cell line not only reversed the SNAI-1 induction but also abrogated the invasiveness caused by STAT3 depletion [15]. In summary, these results demonstrate that STAT3 acts as a molecular adaptor to regulate GSK3 $\beta$ activity, and thus SNAI-1 expression and EMT (Figure 3). It is not clear how STAT3 regulates GSK3 $\beta$ activity, but it may be by acting as a bridge between GSK3 $\beta$ and SNAI-1 to facilitate phosphorylation. In addition, whether the interaction among these three proteins occurs in the cytoplasm or nucleus needs to be investigated. 
Figure 3. STAT3 suppresses EMT by promoting GSK3 $\beta$-mediated phosphorylation and proteasomal degradation of SNAI-1 in IEC. STAT3 inhibits phosphorylation of GSK3 $\beta$ (active), which phosphorylates SNAI-1, leading to ubiquitination and degradation. Consequently, epithelial cell markers, such as claudins, are upregulated and mesenchymal markers, such as vimentin and fibronectin, are down-regulated. When STAT3 is deleted from IEC, unknown environmental cue(s) induce phosphorylation of GSK3 $\beta$ (inactive), most likely via the Akt pathway, leading to accumulation of SNAI and EMT.
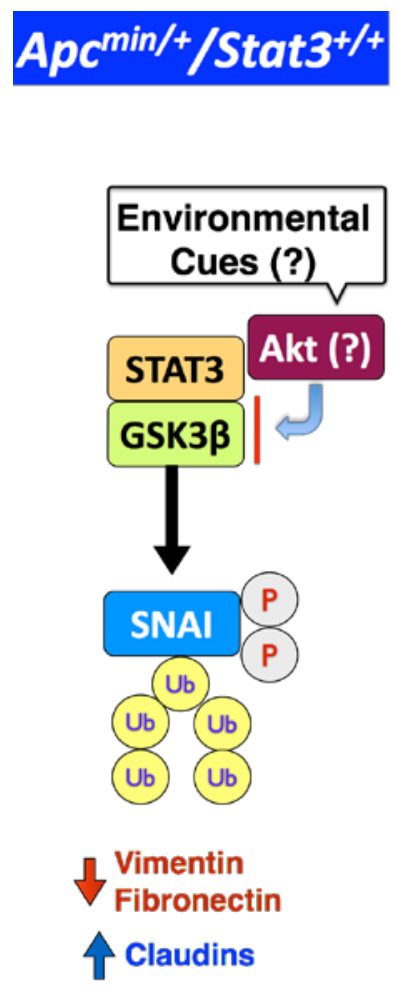

\section{$\mathrm{Apc}^{\mathrm{min} /+} /$ Stat3 $^{-1-}$}

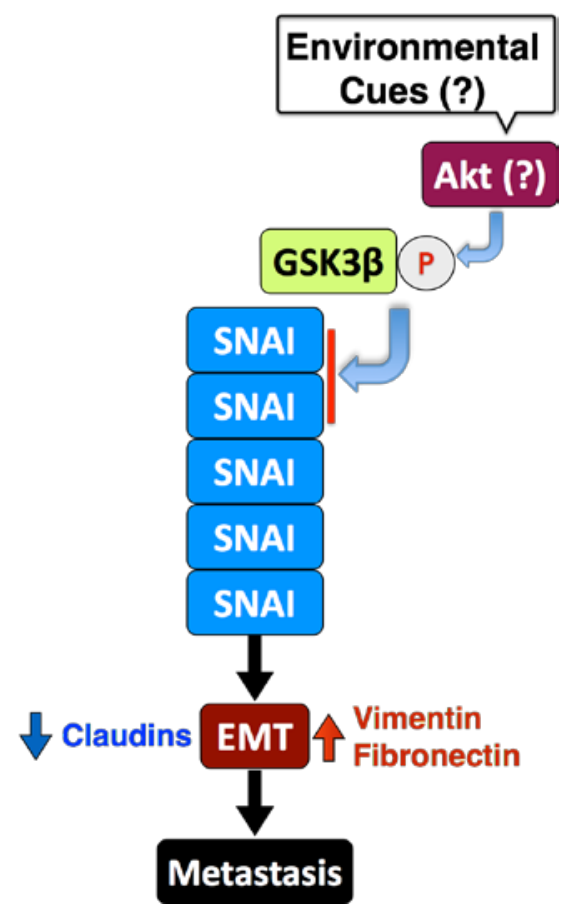

\section{Clinical Implications}

Because of the vast amount of literature supporting the oncogenic role of STAT3, several approaches to inhibit STAT3 activity have been developed for potential clinical trials. These include inhibitors of upstream molecules, such as IL-6 [64] or JAK2 [65,66], and direct inhibition of STAT3 with a STAT3 decoy oligonucleotide [67] or siRNA [68]. While the outcome of these clinical trials should tell us whether STAT3 inhibition is truly efficacious, caution is warranted for CRC trials with direct STAT3 inhibition.

If our model is correct, we should expect adverse effects of direct inhibition of STAT3 in CRC, while targeting upstream molecules such as IL-6 or JAK2 is more complicated, as many targets other than STAT3 could be influenced by these approaches. Another possibility is that the outcome of direct inhibition of STAT3 in CRC is dependent on the underlying genetic or epigenetic background. This becomes increasingly complex when considering the role of STAT3 in non-tumor cells in metastasis (i.e., the tumor microenvironment), as cancer drugs rarely discriminate normal cells from tumor cells. Only further investigations and the outcomes of clinical trials may provide more clarity. 


\section{Conclusions}

Our data and the data from Mesteanu et al. suggest that STAT3 can act as an anti-invasive factor under certain conditions [15,16]. The potential anti-EMT and anti-invasive role of STAT3 warrants rigorous follow-up studies, considering the depth of literature supporting its role as a strong promoter of tumorigenesis and eventual metastasis. Ongoing and future clinical trials on CRC with an agent directly inhibiting STAT3 activity should provide more insight into this paradoxical function of STAT3.

\section{Acknowledgements}

This work was supported by a grant of the Crohn's and Colitis Foundation of America (CCFA) and NIH AI095623 to Eyal Raz.

\section{Author Contributions}

Ji-Hun Mo and Jongdae Lee performed the experiments and Petrus Rudolf de Jong, Alexandra R. Harris, Jongdae Lee and Eyal Raz wrote the manuscript.

\section{Conflicts of Interest}

The authors declare no conflicts of interest.

\section{References}

1. Jemal, A.; Center, M.M.; DeSantis, C.; Ward, E.M. Global patterns of cancer incidence and mortality rates and trends. Cancer Epidemiol. Biomark. Prev. 2010, 19, 1893-1907.

2. Grady, W.M.; Carethers, J.M. Genomic and epigenetic instability in colorectal cancer pathogenesis. Gastroenterology 2008, 135, 1079-1099.

3. Rajagopalan, H.; Nowak, M.A.; Vogelstein, B.; Lengauer, C. The significance of unstable chromosomes in colorectal cancer. Nat. Rev. Cancer 2003, 3, 695-701.

4. Pino, M.S.; Chung, D.C. The chromosomal instability pathway in colon cancer. Gastroenterology 2010, 138, 2059-2072.

5. Lengauer, C.; Kinzler, K.W.; Vogelstein, B. Genetic instability in colorectal cancers. Nature 1997, 386, 623-627.

6. Fensterer, H.; Radlwimmer, B.; Strater, J.; Buchholz, M.; Aust, D.E.; Julie, C.; Radvanyi, F.; Nordlinger, B.; Belluco, C.; van Cutsem, E.; et al. Matrix-comparative genomic hybridization from multicenter formalin-fixed paraffin-embedded colorectal cancer tissue blocks. BMC Cancer 2007, 7, doi:10.1186/1471-2407-7-58.

7. Knutsen, T.; Padilla-Nash, H.M.; Wangsa, D.; Barenboim-Stapleton, L.; Camps, J.; McNeil, N.; Difilippantonio, M.J.; Ried, T. Definitive molecular cytogenetic characterization of 15 colorectal cancer cell lines. Genes Chromosom. Cancer 2010, 49, 204-223.

8. Markowitz, S.D.; Bertagnolli, M.M. Molecular origins of cancer: Molecular basis of colorectal cancer. N. Engl. J. Med. 2009, 361, 2449-2460.

9. Walther, A.; Johnstone, E.; Swanton, C.; Midgley, R.; Tomlinson, I.; Kerr, D. Genetic prognostic and predictive markers in colorectal cancer. Nat. Rev. Cancer 2009, 9, 489-499. 
10. De Sousa, E.M.F.; Wang, X.; Jansen, M.; Fessler, E.; Trinh, A.; de Rooij, L.P.; de Jong, J.H.; de Boer, O.J.; van Leersum, R.; Bijlsma, M.F.; et al. Poor-prognosis colon cancer is defined by a molecularly distinct subtype and develops from serrated precursor lesions. Nat. Med. 2013, 19, 614-618.

11. Sadanandam, A.; Lyssiotis, C.A.; Homicsko, K.; Collisson, E.A.; Gibb, W.J.; Wullschleger, S.; Ostos, L.C.; Lannon, W.A.; Grotzinger, C.; Del Rio, M.; et al. A colorectal cancer classification system that associates cellular phenotype and responses to therapy. Nat. Med. 2013, 19, 619-625.

12. Fearon, E.R.; Vogelstein, B. A genetic model for colorectal tumorigenesis. Cell 1990, 61, 759-767.

13. Atlas, N. Comprehensive molecular characterization of human colon and rectal cancer. Nature 2012, 487, 330-337.

14. Van Cutsem, E.; Nordlinger, B.; Cervantes, A.; Group, E.G.W. Advanced colorectal cancer: ESMO Clinical Practice Guidelines for treatment. Ann. Oncol. 2010, 21, v93-v97.

15. Lee, J.; Kim, J.C.; Lee, S.E.; Quinley, C.; Kim, H.; Herdman, S.; Corr, M.; Raz, E. Signal transducer and activator of transcription 3 (STAT3) protein suppresses adenoma-to-carcinoma transition in Apcmin/+ mice via regulation of Snail-1 (SNAI) protein stability. J. Biol. Chem. 2012, 287, 18182-18189.

16. Musteanu, M.; Blaas, L.; Mair, M.; Schlederer, M.; Bilban, M.; Tauber, S.; Esterbauer, H.; Mueller, M.; Casanova, E.; Kenner, L.; et al. Stat3 is a negative regulator of intestinal tumor progression in Apc(Min) mice. Gastroenterology 2010, 138, doi:10.1053/j.gastro.2009.11.049.

17. Akira, S. Roles of STAT3 defined by tissue-specific gene targeting. Oncogene 2000, 19, 2607-2611.

18. Kane, A.; Deenick, E.K.; Ma, C.S.; Cook, M.C.; Uzel, G.; Tangye, S.G. STAT3 is a central regulator of lymphocyte differentiation and function. Curr. Opin. Immunol. 2014, 28C, 49-57.

19. Kamran, M.Z.; Patil, P.; Gude, R.P. Role of STAT3 in cancer metastasis and translational advances. BioMed Res. Int. 2013, 2013, doi:10.1155/2013/421821.

20. Rebe, C.; Vegran, F.; Berger, H.; Ghiringhelli, F. STAT3 activation: A key factor in tumor immunoescape. Jak-stat 2013, 2, e23010.

21. Yuan, Z.L.; Guan, Y.J.; Chatterjee, D.; Chin, Y.E. Stat3 dimerization regulated by reversible acetylation of a single lysine residue. Science 2005, 307, 269-273.

22. Yang, J.; Chatterjee-Kishore, M.; Staugaitis, S.M.; Nguyen, H.; Schlessinger, K.; Levy, D.E.; Stark, G.R. Novel roles of unphosphorylated STAT3 in oncogenesis and transcriptional regulation. Cancer Res. 2005, 65, 939-947.

23. Yang, J.; Liao, X.; Agarwal, M.K.; Barnes, L.; Auron, P.E.; Stark, G.R. Unphosphorylated STAT3 accumulates in response to IL-6 and activates transcription by binding to NF- $\mathrm{kb}$. Genes Dev. 2007, 21, 1396-1408.

24. Pfeffer, L.M.; Mullersman, J.E.; Pfeffer, S.R.; Murti, A.; Shi, W.; Yang, C.H. STAT3 as an adapter to couple phosphatidylinositol 3-kinase to the IFNAR1 chain of the type I interferon receptor. Science 1997, 276, 1418-1420.

25. Ng, D.C.; Lin, B.H.; Lim, C.P.; Huang, G.; Zhang, T.; Poli, V.; Cao, X. Stat3 regulates microtubules by antagonizing the depolymerization activity of stathmin. J. Cell Biol. 2006, 172, 245-257. 
26. Silver, D.L.; Naora, H.; Liu, J.; Cheng, W.; Montell, D.J. Activated signal transducer and activator of transcription (STAT) 3: Localization in focal adhesions and function in ovarian cancer cell motility. Cancer Res. 2004, 64, 3550-3558.

27. Frank, D.A. STAT3 as a central mediator of neoplastic cellular transformation. Cancer Lett. 2007, 251, 199-210.

28. Bar-Natan, M.; Nelson, E.A.; Xiang, M.; Frank, D.A. STAT signaling in the pathogenesis and treatment of myeloid malignancies. JAK-STAT 2012, 1, 55-64.

29. Kusaba, T.; Nakayama, T.; Yamazumi, K.; Yakata, Y.; Yoshizaki, A.; Nagayasu, T.; Sekine, I. Expression of p-STAT3 in human colorectal adenocarcinoma and adenoma; correlation with clinicopathological factors. J. Clin. Pathol. 2005, 58, 833-838.

30. Morikawa, T.; Baba, Y.; Yamauchi, M.; Kuchiba, A.; Nosho, K.; Shima, K.; Tanaka, N.; Huttenhower, C.; Frank, D.A.; Fuchs, C.S.; et al. STAT3 expression, molecular features, inflammation patterns, and prognosis in a database of 724 colorectal cancers. Clin. Cancer Res. 2011, 17, 1452-1462.

31. Koskela, H.L.; Eldfors, S.; Ellonen, P.; van Adrichem, A.J.; Kuusanmaki, H.; Andersson, E.I.; Lagstrom, S.; Clemente, M.J.; Olson, T.; Jalkanen, S.E.; et al. Somatic STAT3 mutations in large granular lymphocytic leukemia. N. Engl. J. Med. 2012, 366, 1905-1913.

32. Lee, J.; Mo, J.H.; Katakura, K.; Alkalay, I.; Rucker, A.N.; Liu, Y.T.; Lee, H.K.; Shen, C.; Cojocaru, G.; Shenouda, S.; et al. Maintenance of colonic homeostasis by distinctive apical TLR9 signalling in intestinal epithelial cells. Nat. Cell Biol. 2006, 8, 1327-1336.

33. Mellman, I.; Nelson, W.J. Coordinated protein sorting, targeting and distribution in polarized cells. Nat. Rev. Mol. Cell Biol. 2008, 9, 833-845.

34. Tanaka, T.; Soriano, M.A.; Grusby, M.J. SLIM is a nuclear ubiquitin E3 ligase that negatively regulates STAT signaling. Immunity 2005, 22, 729-736.

35. Jarnicki, A.; Putoczki, T.; Ernst, M. Stat3: Linking inflammation to epithelial cancer-More than a "gut" feeling? Cell Div. 2010, 5, doi:10.1186/1747-1028-5-14.

36. Kawada, M.; Seno, H.; Uenoyama, Y.; Sawabu, T.; Kanda, N.; Fukui, H.; Shimahara, Y.; Chiba, T. Signal transducers and activators of transcription 3 activation is involved in nuclear accumulation of beta-catenin in colorectal cancer. Cancer Res. 2006, 66, 2913-2917.

37. Lee, S.H.; Hu, L.L.; Gonzalez-Navajas, J.; Seo, G.S.; Shen, C.; Brick, J.; Herdman, S.; Varki, N.; Corr, M.; Lee, J.; et al. ERK activation drives intestinal tumorigenesis in $\mathrm{Apc}(\mathrm{min} /+)$ mice. Nat. Med. 2010, 16, 665-670.

38. Grivennikov, S.; Karin, E.; Terzic, J.; Mucida, D.; Yu, G.Y.; Vallabhapurapu, S.; Scheller, J.; Rose-John, S.; Cheroutre, H.; Eckmann, L.; et al. IL-6 and Stat3 are required for survival of intestinal epithelial cells and development of colitis-associated cancer. Cancer Cell 2009, 15, 103-113.

39. Liang, J.; Nagahashi, M.; Kim, E.Y.; Harikumar, K.B.; Yamada, A.; Huang, W.C.; Hait, N.C.; Allegood, J.C.; Price, M.M.; Avni, D.; et al. Sphingosine-1-phosphate links persistent STAT3 activation, chronic intestinal inflammation, and development of colitis-associated cancer. Cancer Cell 2013, 23, 107-120.

40. Clapper, M.L.; Cooper, H.S.; Chang, W.C. Dextran sulfate sodium-induced colitis-associated neoplasia: A promising model for the development of chemopreventive interventions. Acta Pharmacol. Sin. 2007, 28, 1450-1459. 
41. Takahashi, M.; Nakatsugi, S.; Sugimura, T.; Wakabayashi, K. Frequent mutations of the beta-catenin gene in mouse colon tumors induced by azoxymethane. Carcinogenesis 2000, 21, 1117-1120.

42. Peinado, H.; Olmeda, D.; Cano, A. Snail, Zeb and bHLH factors in tumour progression: An alliance against the epithelial phenotype? Nat. Rev. Cancer 2007, 7, 415-428.

43. Guo, F.; Parker Kerrigan, B.C.; Yang, D.; Hu, L.; Shmulevich, I.; Sood, A.K.; Xue, F.; Zhang, W. Post-transcriptional regulatory network of epithelial-to-mesenchymal and mesenchymal-to-epithelial transitions. J. Hematol. Oncol. 2014, 7, doi:10.1186/1756-8722-7-19.

44. Tam, W.L.; Weinberg, R.A. The epigenetics of epithelial-mesenchymal plasticity in cancer. Nat. Med. 2013, 19, 1438-1449.

45. Nieto, M.A. Epithelial plasticity: A common theme in embryonic and cancer cells. Science 2013, 342, doi:10.1186/1756-8722-7-19.

46. Oka, H.; Shiozaki, H.; Kobayashi, K.; Inoue, M.; Tahara, H.; Kobayashi, T.; Takatsuka, Y.; Matsuyoshi, N.; Hirano, S.; Takeichi, M.; et al. Expression of E-cadherin cell adhesion molecules in human breast cancer tissues and its relationship to metastasis. Cancer Res. 1993, 53, 1696-1701.

47. Schipper, J.H.; Frixen, U.H.; Behrens, J.; Unger, A.; Jahnke, K.; Birchmeier, W. E-cadherin expression in squamous cell carcinomas of head and neck: Inverse correlation with tumor dedifferentiation and lymph node metastasis. Cancer Res. 1991, 51, 6328-6337.

48. Umbas, R.; Isaacs, W.B.; Bringuier, P.P.; Schaafsma, H.E.; Karthaus, H.F.; Oosterhof, G.O.; Debruyne, F.M.; Schalken, J.A. Decreased E-cadherin expression is associated with poor prognosis in patients with prostate cancer. Cancer Res. 1994, 54, 3929-3933.

49. Yadav, A.; Kumar, B.; Datta, J.; Teknos, T.N.; Kumar, P. IL-6 promotes head and neck tumor metastasis by inducing epithelial-mesenchymal transition via the JAK-STAT3-SNAIL signaling pathway. Mol. Cancer Res. 2011, 9, 1658-1667.

50. Xie, T.X.; Huang, F.J.; Aldape, K.D.; Kang, S.H.; Liu, M.; Gershenwald, J.E.; Xie, K.; Sawaya, R.; Huang, S. Activation of Stat3 in human melanoma promotes brain metastasis. Cancer Res. 2006, 66, 3188-3196.

51. Lee, J.; Mo, J.H.; Raz, E. Department of Medicine, University of California, San Diego, CA, USA. Unpublished data, 2014.

52. Chen, X.; Halberg, R.B.; Burch, R.P.; Dove, W.F. Intestinal adenomagenesis involves core molecular signatures of the epithelial-mesenchymal transition. J. Mol. Histol. 2008, 39, 283-294.

53. Tsareva, S.A.; Moriggl, R.; Corvinus, F.M.; Wiederanders, B.; Schutz, A.; Kovacic, B.; Friedrich, K. Signal transducer and activator of transcription 3 activation promotes invasive growth of colon carcinomas through matrix metalloproteinase induction. Neoplasia 2007, 9, 279-291.

54. Ota, I.; Li, X.Y.; Hu, Y.; Weiss, S.J. Induction of a MT1-MMP and MT2-MMP-dependent basement membrane transmigration program in cancer cells by Snail1. Proc. Natl. Acad. Sci. USA 2009, 106, 20318-20323.

55. Hotary, K.B.; Allen, E.D.; Brooks, P.C.; Datta, N.S.; Long, M.W.; Weiss, S.J. Membrane type I matrix metalloproteinase usurps tumor growth control imposed by the three-dimensional extracellular matrix. Cell 2003, 114, 33-45. 
56. Kanazawa, A.; Oshima, T.; Yoshihara, K.; Tamura, S.; Yamada, T.; Inagaki, D.; Sato, T.; Yamamoto, N.; Shiozawa, M.; Morinaga, S.; et al. Relation of MT1-MMP gene expression to outcomes in colorectal cancer. J. Surg. Oncol. 2010, 102, 571-575.

57. Zucker, S.; Cao, J. Selective matrix metalloproteinase (MMP) inhibitors in cancer therapy: Ready for prime time? Cancer Biol. Ther. 2009, 8, 2371-2373.

58. Xiong, H.; Hong, J.; Du, W.; Lin, Y.W.; Ren, L.L.; Wang, Y.C.; Su, W.Y.; Wang, J.L.; Cui, Y.; Wang, Z.H.; et al. Roles of STAT3 and ZEB1 proteins in E-cadherin down-regulation and human colorectal cancer epithelial-mesenchymal transition. J. Biol. Chem. 2012, 287, 5819-5832.

59. Zhou, C.; Tong, Y.; Wawrowsky, K.; Melmed, S. PTTG acts as a STAT3 target gene for colorectal cancer cell growth and motility. Oncogene 2014, 33, 851-861.

60. Calon, A.; Espinet, E.; Palomo-Ponce, S.; Tauriello, D.V.; Iglesias, M.; Cespedes, M.V.; Sevillano, M.; Nadal, C.; Jung, P.; Zhang, X.H.; et al. Dependency of colorectal cancer on a TGF-beta-driven program in stromal cells for metastasis initiation. Cancer Cell 2012, 22, 571-584.

61. Zhou, B.P.; Deng, J.; Xia, W.; Xu, J.; Li, Y.M.; Gunduz, M.; Hung, M.C. Dual regulation of Snail by GSK-3beta-mediated phosphorylation in control of epithelial-mesenchymal transition. Nat. Cell Biol. 2004, 6, 931-940.

62. Cross, D.A.; Alessi, D.R.; Cohen, P.; Andjelkovich, M.; Hemmings, B.A. Inhibition of glycogen synthase kinase-3 by insulin mediated by protein kinase B. Nature 1995, 378, 785-789.

63. Doble, B.W.; Patel, S.; Wood, G.A.; Kockeritz, L.K.; Woodgett, J.R. Functional redundancy of GSK-3 $\alpha$ and GSK-3 $\beta$ in Wnt/beta-catenin signaling shown by using an allelic series of embryonic stem cell lines. Dev. Cell 2007, 12, 957-971.

64. Waldner, M.J.; Foersch, S.; Neurath, M.F. Interleukin-6-A key regulator of colorectal cancer development. Int. J. Biol. Sci. 2012, 8, 1248-1253.

65. Senft, C.; Priester, M.; Polacin, M.; Schroder, K.; Seifert, V.; Kogel, D.; Weissenberger, J. Inhibition of the JAK-2/STAT3 signaling pathway impedes the migratory and invasive potential of human glioblastoma cells. J. Neuro-Oncol. 2011, 101, 393-403.

66. Sai, K.; Wang, S.; Balasubramaniyan, V.; Conrad, C.; Lang, F.F.; Aldape, K.; Szymanski, S.; Fokt, I.; Dasgupta, A.; Madden, T.; et al. Induction of cell-cycle arrest and apoptosis in glioblastoma stem-like cells by WP1193, a novel small molecule inhibitor of the JAK2/STAT3 pathway. J. Neuro-Oncol. 2012, 107, 487-501.

67. Sen, M.; Thomas, S.M.; Kim, S.; Yeh, J.I.; Ferris, R.L.; Johnson, J.T.; Duvvuri, U.; Lee, J.; Sahu, N.; Joyce, S.; et al. First-in-human trial of a STAT3 decoy oligonucleotide in head and neck tumors: Implications for cancer therapy. Cancer Discov. 2012, 2, 694-705.

68. Li, G.H.; Wei, H.; Chen, Z.T.; Lv, S.Q.; Yin, C.L.; Wang, D.L. STAT3 silencing with lentivirus inhibits growth and induces apoptosis and differentiation of U251 cells. J. Neuro-Oncol. 2009, 91, 165-174.

(C) 2014 by the authors; licensee MDPI, Basel, Switzerland. This article is an open access article distributed under the terms and conditions of the Creative Commons Attribution license (http://creativecommons.org/licenses/by/3.0/). 